\title{
Abdominal Textiloma, 10 Years after an Appendectomy: Case Report
}

Faouzi Laytimi*, Ahmed Oubihi, Mohamed Fdil, Ouadii Mouaqit, El Bachir Benjelloun, Abdelmalek Ousadden, Khalid Ait Taleb, Hicham El Bouhaddouti

Faculty of Medicine and Pharmacy, Sidi Mohamed Ben Abdellah University of Fez, Morocco

Visceral Surgery Department A, CHU Hassan II, Fez, Morocco

DOI: $10.36347 /$ sasjs.2021.v07i03.009

| Received: 02.03.2021 | Accepted: 20.03.2021 | Published: 23.03.2021

*Corresponding author: Faouzi Laytimi

\section{Abstract}

Textiloma commonly refers to a foreign textile body (compress or surgical field), left involuntarily during a surgical intervention. Its preoperative diagnosis remains difficult due to the absence of pathognomonic clinical and radiological signs. It is a surgical complication which represents a typical example of medical malpractice, the exact incidence of which is difficult to determine due to possible legal repercussions, hence the need for a systematic approach to the organization of the operating room and the implementation of strict textile counting protocols in order to avoid this type of incident. We report a case of a textiloma that developed 10 years after an appendectomy.

Keywords: Textiloma, medical malpractice, textile, counting.

Copyright $\left({ }_{0} 2021\right.$ The Author(s): This is an open-access article distributed under the terms of the Creative Commons Attribution 4.0 International License (CC BY-NC 4.0) which permits unrestricted use, distribution, and reproduction in any medium for non-commercial use provided the original author and source are credited.

\section{INTRODUCTION}

Textiloma, or gossybipoma (derived from 'gossypium' meaning 'cotton' in Latin and 'boma' meaning 'place of hiding' in Swahili), is an inflammatory granuloma centered by textile fibers (compress or field surgical) forgotten at the level of an operative site $[1,4]$. It is an extremely rare postoperative complication predominantly in the abdomino-pelvic region [2], and is one of the most publicized medical errors. Reporting to us the case of a textiloma revealed 10 years after an appendectomy.

\section{PATIENT AND OBSERVATION}

We report the case of a 50-year-old patient with an antecedent of appendectomy 10 years ago, the postoperative effects were simple, and the pathological study supporting lymphoid hyperplasia of the appendix; Admitted for pain in the right iliac fossa progressing in flares for a few months, without other associated signs, in which the physical examination revealed a scar from McBurney-type laparotomy and tenderness of the right iliac fossa. He initially benefited from a biological assessment returning to normal, in particular no inflammatory syndrome, then an abdominal ultrasound objectifying a right pelvic solido-cystic mass, containing calcifications of well-circumscribed oval shape, measuring $35^{*} 32 \mathrm{~mm}$ not vascularized in the study doppler, associated with a slight dilation of the right urinary tract (Figure 1). To better characterize this ultrasound image, an abdomino-pelvic tomodensitometry was performed showing a cystic formation with a tissue contingent, oval $44 * 36 * 45 \mathrm{~mm}$ not enhancing after injection of the contrast product, also containing images of calcium density practically metallic, making the eventuality of a textiloma very likely (Figure 2).

The Surgical exploration revealed a mass of about $40 \mathrm{~mm}$ in diameter at the level of the right iliac fossa of hard consistency, adherent to the last ileal loop and the cecum, after its release from its intercourse, its opening revealed that it is a compress marked by a radiopaque thread (Figure 3), thus confirming the diagnosis of a textiloma, the operative consequences were simple, and the patient was discharged from the hospital 2 days after surgery. 


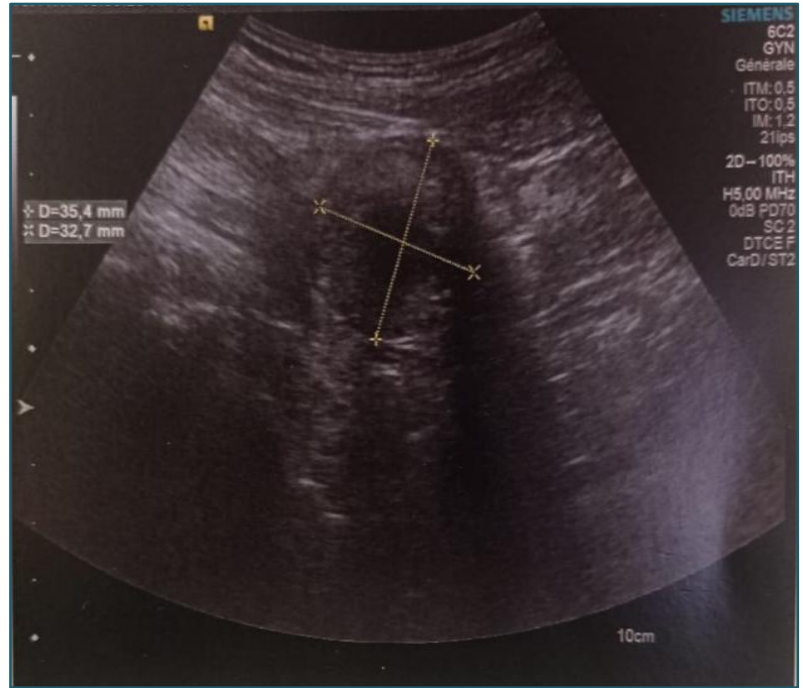

Fig-1: Ultrasound image showing a right pelvic solido-cystic mass of well circumscribed non-specific oval shape

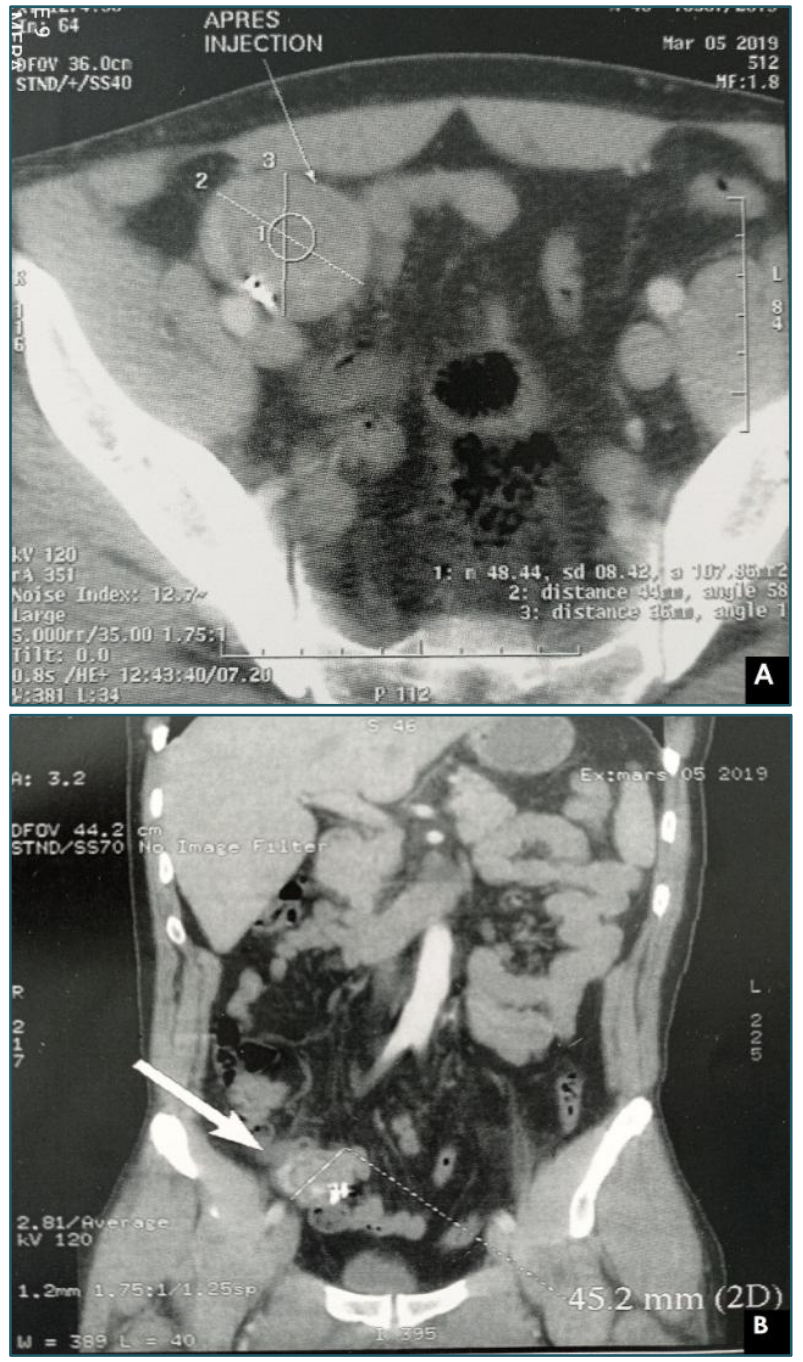

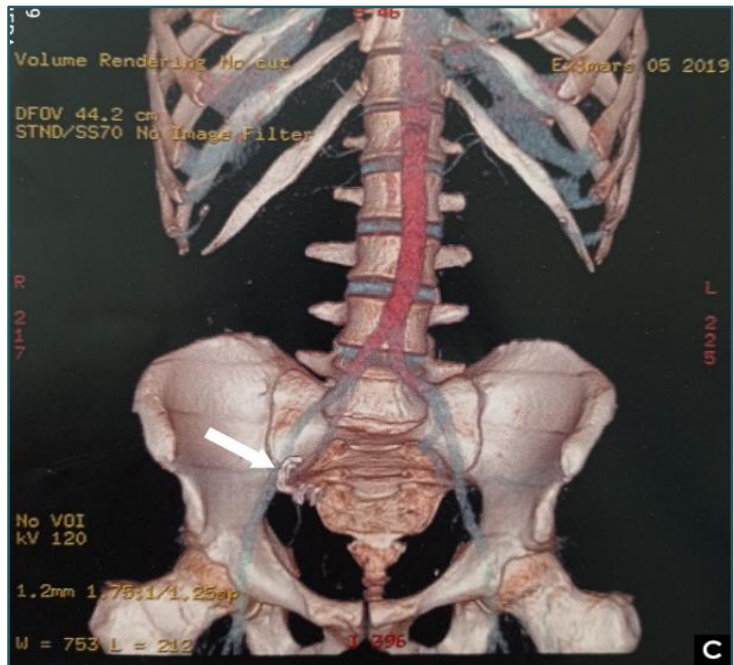

Fig-2: A-B, cystic formation with a contingent tissue, not enhancing after injection of the product of contrast, also containing images of almost metallic calcium density; C, 3D reconstruction showing the foreign body
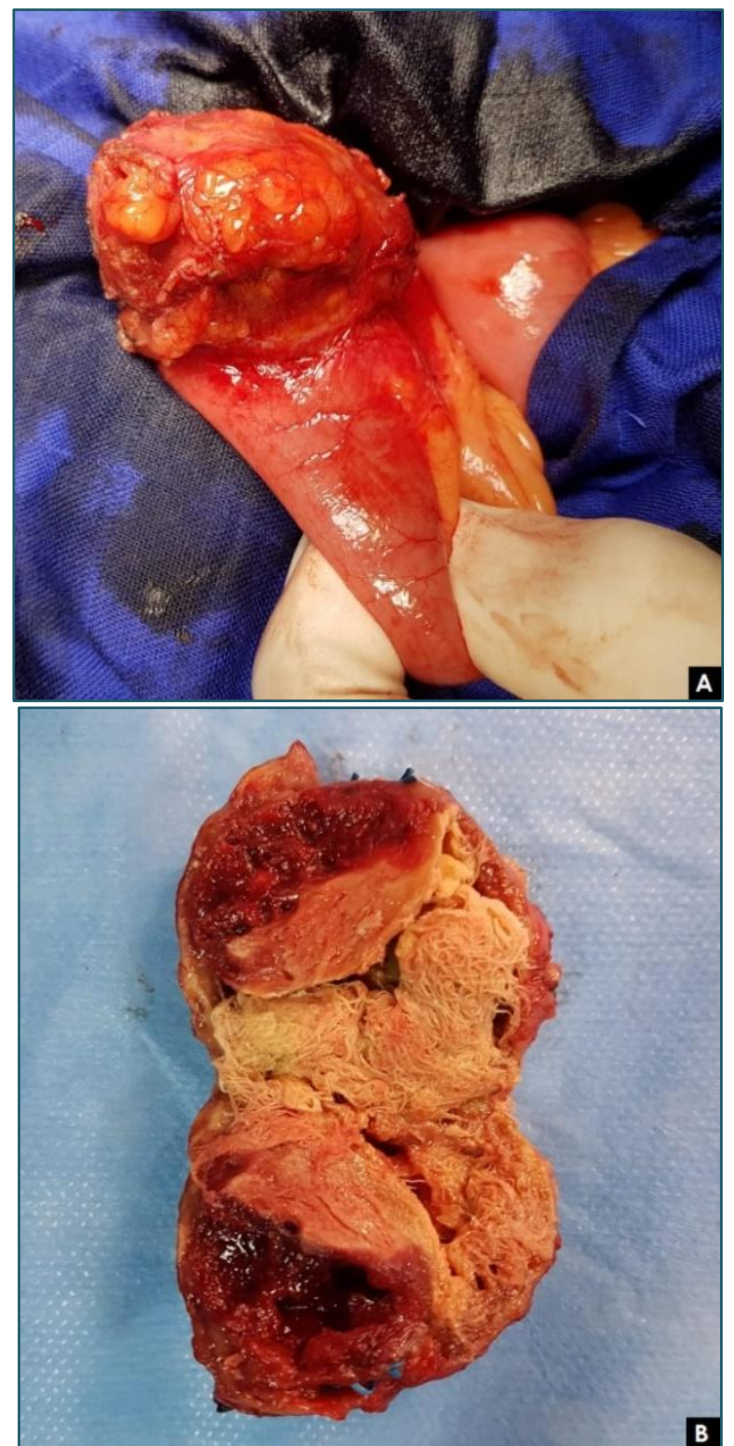

Fig-3: A) Operative image showing the mass of the right iliac fossa which is adherent to the last ileal loop and the cecum; B) The opening of the operating mass revealed the compress marked with a radiopaque wire 


\section{DISCUSSION}

Textilloma is a fear of all surgeons, which can lead to forensic proceedings, and is responsible for multiple complications. Its incidence is not known but it is reported between $1 / 100$ and $1 / 3000$ surgical operations and between 1/1000 and 1/1500 abdominal surgeries [3], the first case of which was reported by Wilson in 1884, and the literature only has isolated cases and short series [4]. The risk factors are presented mainly by an emergency surgical procedure, an unexpected change of procedure, obesity, as well as the intervention of more than one surgical team, the interventions during the second half of the night, the losses blood levels and the absence of counting of textiles and instruments [5].

The textile forgotten intraperitoneally remains free for the first 24 hours, then its fibers cause an inflammatory reaction with exudation known as 'gossypidoma', which creates adhesions with the surrounding tissues and generates the constitution of an inflammatory granuloma towards the 8th day and fibrosis is organized from the 13th day. This development explains, in the absence of infection, the possibilities of encystment or even calcifications with a sometimes long tolerance as in our observation $[6,7]$. The infection of the foreign body is expressed by infectious clinical signs allowing early diagnosis, otherwise the inflammatory process progresses to encystment or even calcification with sometimes very long tolerance. The course of textiloma is unpredictable $[8,9]$. Thus, its elimination can be by fistulization to the skin or hollow organs, and migrations have been described through the diaphragm, the intestinal wall with obstruction, the vagina [10].

This disease can progress insidiously, with late discovery, several years after the initial intervention, accidentally or in a picture of "tumor syndrome" [2]. What makes the history and the notion of previous surgery an essential element for the diagnosis given the poor physical examination, it associated chronic transit disorders with intermittent abdominal pain, with an inconsistent palpable abdominal mass, which makes it essential to resort to paraclinical examinations:

- X-ray of the Abdomen without Preparation: lacks specificity and is non-contributory, as often in sub-occlusive syndromes. This accounts for the interest of the use of marked compresses [1].

- The ultrasound makes it possible to objectify a non-specific pseudo-tumoral aspect, however in case of encystment it can show a hyperechoic contingent containing air bubbles, calcifications which are inconstant and the posterior shadow cone are very suggestive in the context of postoperative consequences [1].
- Computed tomography, or even MRI for some teams, is the examination of choice for the preoperative diagnosis of textiloma as it allows a complete exploration of the abdominal cavity in search of complications (fistulas, pneumoperitoneum, abscess). The characteristic image is a spongiform appearance with the presence of small air bubbles [1], calcifications or even an image of metallic density, as in our case. Textiloma can be confused with a digestive tumor (lymphoma, colonic adenocarcinoma, etc.) [11].

Surgical exploration remains the only way to confirm the diagnosis and treat the disease. Extraction of the foreign body can be easy in the early postoperative stages. However, the later discovery makes the release of the mass more laborious with the possibility of iatrogenic perforations which can lead to intestinal resections [10].

The real treatment lies in the establishment of means of prevention represented mainly by the counting of compresses and fields by the operating team at the beginning and at the end of the intervention, which remains an effective but still insufficient means. As well as the use of radiopaque labeled compresses proposed by CROSSEN and al in 1940 [11, 12], which allows an X-ray to be taken on the operating table in case of doubt.

\section{CONCLUSION}

The risk of forgetting textiles must always be taken into consideration with each surgical intervention, as it can lead to serious complications, as well as medico-legal proceedings involving the responsibility of the operating team. This should be the subject of awareness-raising, emphasizing caution and taking preventive measures seriously.

Conflicts of interest: The authors declare that they have no conflicts of interest.

\section{REFEFERENCES}

1. O'Connor AR, Coakley FV, Meng MV, Eberhardt SC. Imaging of retained surgical sponges in the abdomen and pelvis .AJR Am J Roentgenol. 2003 Feb;180(2):481-9.

2. Doha K, Thiam I, Takin R.C.A, Bissiroub I, Woto Gaye G. A case of renal gossypibomas mimicking tumor pathological discovery. Afr J Urol. 2017; 23:364-367.

3. Sarda AK, Pandey D, Neogi S and Dhir U. Postoperative complications due to a retained surgical sponge. Singapore Med J. 2007;48(6):160164.

4. Chopra S, Suri V, Sikka P, Aggarwal N. A case series on gossypiboma - varied clinical presentations and their management. J ClinDiagn Res. 2015 Dec; 9(12): QR01-QR03.

5. Gawande AA, Studdert DM, Orav EJ, Troyen J, Brennan M, Zinner M. Risk factors for retained 
instruments and sponges after surgery. N Engl J Med. 2003; 348:229-235.

6. Chambi I, Tasker RR, Gentili F, Lougheed WM, Smyth HS, Marshall J, Young I, Deck J, Shrubb J. Gauze-induced granuloma (gauzoma): an uncommon complication of gauze reinforcement of berry aneurysms. J Neurosurg. 1990 Feb;72(2):163-70.

7. Saadi A, Bouzouita A, Kerkeni W, Ayed H, Ben Miled A. An abdominal mass. Rev med interne. 2017; 38:347-348.

8. Braude P. Encysted gossypidoma of the lesser omuntum: complementary aspect of echography and CI.J BelgeRadiol 1992;75:125 -8.
9. Haji A, Abbar M, Bouchama R, Moulay A. Les textilomesintrapéritonéaux: à propos de 5 cas. J Chir. 1988; 125:353-7.

10. Sarr ISS, Ndong A, Thiam O, Seck M, Touré AO. Intraluminal digestive textiloma under multiple clinical aspects. J afr chir digest. 2018;18(2);25742578.

11. Erguibi D, Robleh H.F, Ajbal M, Kadiri B. Abdominal textiloma, about a case. Pan Afr Med J. 2015; 21: 244.

12. Crossen HS, Crossen DF. Foreign bodies left in the abdomen. CV Mosby Co St Louis; 1940. 